\title{
In silico characterization of hypothetical proteins from Paracoccidioides lutzii
}

\author{
P.F.F. Silva', E. Novaes², M. Pereira ${ }^{1}$, C.M.A. Soares ${ }^{1}$, C.L. Borges ${ }^{1}$ and \\ S.M. Salem-Izacc ${ }^{1}$ \\ 1Departamento de Bioquímica e Biologia Molecular, Universidade Federal de Goiás, \\ Goiânia, GO, Brasil \\ ${ }^{2}$ Escola de Agronomia, Universidade Federal de Goiás, Goiânia, GO, Brasil \\ Corresponding author: S.M. Salem-Izacc \\ E-mail: silviaizacc@gmail.com
}

Genet. Mol. Res. 14 (4): 17416-17425 (2015)

Received August 27, 2015

Accepted October 21, 2015

Published December 21, 2015

DOI http://dx.doi.org/10.4238/2015.December.21.11

ABSTRACT. Nearly $60 \%$ of Paracoccidioides lutzii genes encode products annotated as hypothetical or predicted proteins (HPs). In this study, we describe the global detection and functional inference of HPs, using computational methods based on sequence similarity, identification of targeting signals, presence of known protein domains, and use of the Gene Ontology functional classification scheme. Our analysis enabled a highthroughput characterization of predicted cellular localization and presence of protein domains, clustering HPs into different functional categories including metabolism, localization, cell cycle, response to stimulus, and signaling. To investigate $P$. lutzii HP expression profiles, we used data obtained from the expressed sequence tag database (dbEST). These analyses revealed $2364 \mathrm{HPs}$ expressed in different situations, namely in mycelial and yeast forms, during the transition from mycelium to yeast, and under conditions mimicking infection. Based on this transcriptomic data, we performed a functional enrichment analysis according to the domains present in the HPs expressed in each condition. The most overrepresented functional domains were those involved in the regulation of gene expression, suggesting important and as yet undescribed roles 
for these HPs in the adaptation of $P$. lutzii to environmental conditions. In addition, the expression profiles of six randomly selected HPs were analyzed by quantitative real-time polymerase chain reaction in order to verify their expression in the complementary DNA libraries analyzed in this investigation. The approach used in this study should improve functional characterization of $P$. lutzii HPs.

Key words: Paracoccidioides; Hypothetical proteins; In silico analysis

\section{INTRODUCTION}

Despite the great number of genomic and transcriptomic sequences deposited in databases over recent years, a large proportion of the genes identified encode a group of proteins with unknown biochemical, biophysical, or cellular functions, annotated as predicted or hypothetical proteins (HPs). HPs are proteins predicted from nucleic acid sequences, based on computational identification of gene structure, but for which no evidence of in vivo expression exists. These proteins may represent up to half of the proteome for several organisms (Loewenstein et al., 2009; Shahbaaz et al., 2013; ljaq et al., 2015).

Paracoccidioides brasiliensis and $P$. lutzii are thermodimorphic pathogens that cause paracoccidioidomycosis, a mycosis endemic to Latin America. The genus Paracoccidioides comprises the cryptic species $P$. brasiliensis S1, PS2, and PS3, and the closely related $P$. lutzii. Until recently, $P$. brasiliensis and $P$. lutzii were considered a single species, identified using the former's name (Matute et al., 2006; Teixeira et al., 2009). Infection results from inhalation of hyphal fragments or spores released from mycelium $(M)$, resulting in exposure to elevated temperatures and triggering a morphological switch to the yeast (Y) form (Restrepo, 1985; Franco et al., 1987). The pathogenicity of these fungi seems to be related to this morphological change, since strains unable to differentiate are often avirulent (Medoff et al., 1987; Borges-Walmsley et al., 2002).

Nearly $60 \%$ of Paracoccidioides genes are annotated as encoding HPs (Desjardins et al., 2011). The characterization of proteins of unknown function poses a challenge both to functional genomics and to biology in general, and if achieved, could help to fill the gaps in attempts to attain complete genomic and proteomic data. Several computational approaches are available for predicting protein function (Doerks et al., 2012; Qiao et al., 2013; ljaq et al., 2015). This work describes an in silico characterization of unclassified proteins from $P$. lutzii using analysis of predicted cellular localization, conserved protein domains, and functional classification according to the Gene Ontology (GO) scheme. In addition, we measured the expression of HPs in complementary DNA (cDNA) libraries constructed from P. brasiliensis and P. lutzii M and Y forms (Goldman et al., 2003; Felipe et al., 2005), during the transition from $M$ to $Y$ (Bastos et al., 2007), and under conditions mimicking infection (Bailão et al., 2006; Bailão et al., 2007; Costa et al., 2007). We then performed functional enrichment analysis of the HPs expressed in these different transcriptomes to identify overrepresented protein domains.

\section{MATERIAL AND METHODS}

\section{Determination of Paracoccidioides HPs}

A list of HPs was obtained from the P. brasiliensis genome database (http://www.broadin- 
stitute.org/annotation/genome/paracoccidioides_brasiliensis/MultiDownloads.html). For the present study, HPs were considered as all proteins annotated as "hypothetical protein", "conserved hypothetical protein", or "predicted protein".

\section{Identification of HPs expressed in Paracoccidioides cDNA libraries}

To compile a list of expressed HPs, we extracted all Paracoccidioides expressed sequence tags (ESTs) deposited in the dbEST database (http://www.ncbi.nlm.nih.gov/dbEST) as of September 19, 2013. These ESTs were then used as queries in blastx searches (Altschul et al., 1990) against the $P$. lutzii protein database available on the Broad Institute website (Desjardins et al., 2011). The following cutoff values were used to identify expressed HPs: E-value, 10-6; identity, $60 \%$; and alignment length, 50 amino acids.

\section{Prediction of cellular localization}

Cellular localization of HPs was predicted using TargetP (Emanuelsson et al., 2007), under the default settings. TargetP suggests the subcellular location of eukaryotic proteins based on the predicted presence of $\mathrm{N}$-terminal presequences.

\section{GO term and protein domain annotation}

We assigned GO terms and protein domains to HPs encoded in the $P$. lutzii genome using the Blast2GO program (Conesa et al., 2005). Deduced HP sequences were compared to those in the National Center for Biotechnology Information (NCBI) non-redundant protein database, with a cutoff value of $10^{-6}$. The pipeline began with a BLAST search against this database, following which the program consolidated annotations of BLAST hits to attribute a putative function to each protein based on GO data.

\section{Enrichment analysis for protein domains}

In order to assign a biological meaning to the HPs expressed in the different CDNA libraries, we performed an enrichment analysis of the protein domains identified in each condition. This was carried out online by inserting the list of expressed HP locus IDs for each condition into the Broad Institute's functional enrichment analysis tool (http://www.broadinstitute.org/ annotation/genome/paracoccidioides_brasiliensis/BatchSelect.html?target=GeneEnrichment. $\mathrm{html})$. We imposed a corrected $\mathrm{P}$ value cutoff of 0.2 .

\section{P. lutzii growth conditions}

P. lutzii strain Pb01 (ATCC MYA-826) was used in quantitative real-time polymerase chain reaction (qPCR) experiments in this study. Cells were cultured in liquid Fava Netto's medium $(0.3 \%$ proteose peptone, $1 \%$ peptone, $0.5 \%$ meat extract, $0.5 \%$ yeast extract, $4 \%$ glucose, and $0.5 \% \mathrm{NaCl}, \mathrm{pH} 7.2$ ) at 36 and $22^{\circ} \mathrm{C}$, for $\mathrm{Y}$ and $\mathrm{M}$ forms, respectively. The $\mathrm{M}$ to $\mathrm{Y}$ transition was performed in the same medium by raising the incubation temperature from $22^{\circ}$ to $36^{\circ} \mathrm{C}$ over $22 \mathrm{~h}$. 


\section{Incubation of $P$. lutzii Y cells with human blood or plasma}

P. lutzii Y cells were incubated with either human blood or plasma, as described by Bailão et al. (2006, 2007). Briefly, human blood from five healthy donors was collected by venipuncture using heparinized syringes and centrifuged at $1000 \mathrm{~g}$. Fungal cells were harvested from 7-day-old cultures and washed once with phosphate buffered saline (PBS; $137 \mathrm{mM} \mathrm{NaCl}, 2.7 \mathrm{mM} \mathrm{KCl}, 1.4$ $\mathrm{mM} \mathrm{NaH}_{2} \mathrm{PO}_{4}, 4.3 \mathrm{mM} \mathrm{Na}_{2} \mathrm{HPO}_{4}, \mathrm{pH} 7.4$ ) before being inoculated (at $5 \times 10^{6}$ cells $/ \mathrm{mL}$ ) into $5 \mathrm{~mL}$ of human blood or plasma at $36^{\circ} \mathrm{C}$ for $10 \mathrm{~min}$ with shaking. They were then collected by centrifugation for $5 \mathrm{~min}$ at $1500 \mathrm{~g}$, and washed five times with PBS. The use of human blood was approved by the Research Ethics Committee of Universidade Federal de Goiás. Written informed consent was obtained from all donors. All specimens were handled and made anonymous according to ethical and legal standards.

\section{RNA extraction}

Total RNA was extracted using TRIzol reagent (Invitrogen, Carlsbad, CA, USA) following the manufacturer protocol. RNA samples were isolated from $Y$ and $M$ cells, those undergoing $M$ to $Y$ differentiation, and $Y$ cells having been incubated with human blood or plasma for 10 min.

\section{qPCR assay}

RNA samples were reverse transcribed using a High Capacity RNA-to-cDNA kit (Applied Biosystems, Foster City, CA, USA). cDNA samples were then diluted 1:5 in water, and qPCR was performed using SYBR green PCR master mix and the StepOnePlus Real-Time PCR System (both Applied Biosystems). Specific primers (Table S1) were designed for each selected gene using the NCBI Primer-BLAST program (http://www.ncbi.nlm.nih.gov/tools/primer-blast/). Reaction specificity was evaluated by analyzing the dissociation curves of amplified products. QPCR was performed in triplicate for each cDNA sample. Measurements were normalized using Paracoccidioides beta-tubulin as an endogenous control and relative transcript expression was estimated with the comparative cycle threshold $(\Delta \Delta \mathrm{Ct})$ method (Livak and Schmittgen, 2001).

\section{RESULTS AND DISCUSSION}

\section{Overview of HPs encoded in the Paracoccidioides genome and transcriptomes}

Annotation of the genomes of three Paracoccidioides strains revealed that approximately $60 \%$ of their genes encode proteins of unknown function, generally annotated as HPs. Almost $50 \%$ of these HPs are conserved, i.e. they have also been identified in other organisms, while the remainder appear to be restricted to Paracoccidioides (Table 1).

We also utilized the Paracoccidioides ESTs deposited in the dbEST. Our analysis revealed that the percentage of peptide-encoding transcripts identified as HPs in the cDNA libraries ranged from 24 to $46 \%$ (Table 2), indicating that a substantial number of HPs are expressed. Altogether, we detected 2364 unique transcripts annotated as HPs in at least one of the libraries. In an effort to attribute biological meaning to the expression data, the libraries were arranged into three conditions: "M/Y", M and Y cells (Goldman et al., 2003; Felipe et al., 2005); "transition", cells undergoing 
morphological transition from $\mathrm{M}$ to $\mathrm{Y}$ form (Bastos et al., 2007); and "infection", cells under conditions mimicking infection (Bailão et al., 2006, 2007; Costa et al., 2007). Of the 2364 unique HP transcripts, 2324 were able to be placed into one or more of these three conditions. Figure 1 shows a Venn diagram depicting the number of HPs identified in each situation. Only 25 were expressed under all conditions, demonstrating that these proteins are regulated by environmental factors. Interestingly, 117 HPs were exclusively expressed in an infection-like context, indicating important roles during this process, and emphasizing the need to establish their functions.

Table 1. Summary of the proteins annotated as hypothetical encoded in the genomes of Paracoccidioides species
and strains.
\begin{tabular}{lccccc}
\hline Species (strain) & Total proteins & $\begin{array}{c}\text { Number of predicted } \\
\text { proteins (\%) }\end{array}$ & $\begin{array}{c}\text { Number of hypothetical } \\
\text { proteins (\%) }\end{array}$ & $\begin{array}{c}\text { Number of conserved } \\
\text { hypothetical proteins (\%) }\end{array}$ & $\begin{array}{c}\text { Total number } \\
\text { of HPs (\%) }\end{array}$ \\
\hline P. lutzii (Pb01) & 9132 & $2116(23.2 \%)$ & $592(6.5 \%)$ & $2928(32.1 \%)$ & $5636(61.7 \%)$ \\
P. brasiliensis (Pb03) & 7876 & $1922(24.4 \%)$ & $280(3.6 \%)$ & $2540(32.2 \%)$ & $4742(60.2 \%)$ \\
P. brasiliensis (Pb18) & 8741 & $1997(22.8 \%)$ & $401(4.6 \%)$ & $2916(33.4 \%)$ & $5314(60.8 \%)$ \\
\hline
\end{tabular}

$\mathrm{HP}=$ hypothetical protein.

Table 2. Survey of the hypothetical protein transcripts expressed in Paracoccidioides transcriptomes.

\begin{tabular}{llcrrr}
\hline Condition & Library ID (cells from which mRNA was extracted) & Strain & $\begin{array}{c}\text { Number of ESTs } \\
\text { in the library }\end{array}$ & $\begin{array}{c}\text { Number of peptides } \\
\text { with a blastx hit }\end{array}$ & $\begin{array}{c}\text { Number of HP peptides } \\
\text { with a blastx hit* }\end{array}$ \\
\hline M/Y & aLIBEST_012137 (M and Y) & Pb01 & 19,898 & 4075 & $1872(45.94 \%)$ \\
& bLIBEST_010891 (Y) & $\mathrm{Pb} 18$ & 13,490 & 1868 & $715(38.28 \%)$ \\
Transition & 'LIBEST_020653 (22 h of M $\rightarrow$ Y transition) & $\mathrm{Pb01}$ & 1107 & 477 & $176(36.90 \%)$ \\
Infection & 'LIBEST_020789 (Y recovered from the livers of infected mice) & $\mathrm{Pb01}$ & 4934 & 1019 & $408(40.04 \%)$ \\
& eLIBEST_019382 (Y incubated with human blood or plasma) & $\mathrm{Pb01}$ & 1486 & 151 & $36(23.84 \%)$ \\
- & All five libraries & - & 40,915 & 5012 & $2364(47.17 \%)$ \\
\hline
\end{tabular}

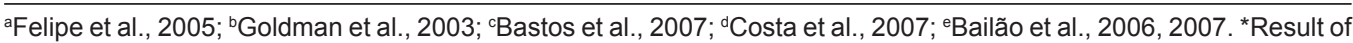
a blastx search against the Paracoccidioides protein database. $\mathrm{M}=$ mycelium; $\mathrm{Y}=$ yeast; $\mathrm{EST}=$ expressed sequence tag; HP = hypothetical protein.

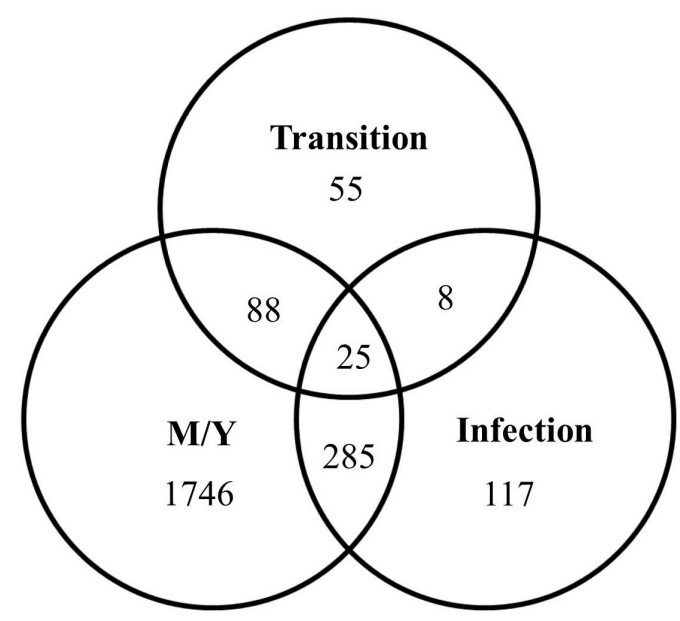

Figure 1. Venn diagram representing the number of hypothetical proteins expressed in each condition. $M=$ mycelium; $\mathrm{Y}=$ yeast. 


\section{Protein length distribution}

All HPs and annotated proteins encoded in the $P$. lutzii genome were plotted according to their length, and the percentage of expressed HPs in each length category was determined. As shown in Figure 2, more than $90 \%$ of the proteins consisting of 1 to 100 amino acid residues were annotated as HPs, however, only $11 \%$ of these had corresponding transcripts in the cDNA libraries. Of those proteins between 201 and 300 amino acids in length, more than $70 \%$ were identified as HPs, but only $31 \%$ of these were expressed. In contrast, the percentage of the largest proteins (longer than 301 residues) annotated as HPs was much lower (between 36 and 58\%). However, a much larger proportion of these, from 47 to $67 \%$, were expressed. These results suggest that most of the small proteins annotated as HPs may in fact correspond to incorrectly annotated genes and their legitimacy needs further confirmation. Our data corroborates earlier findings that open reading frame (ORF) calls relating to small peptides may simply indicate random sequences coincidentally forming ORFs (Qiao et al., 2013). These small sequences might also represent ancient functional genes having undergone pseudogenization.

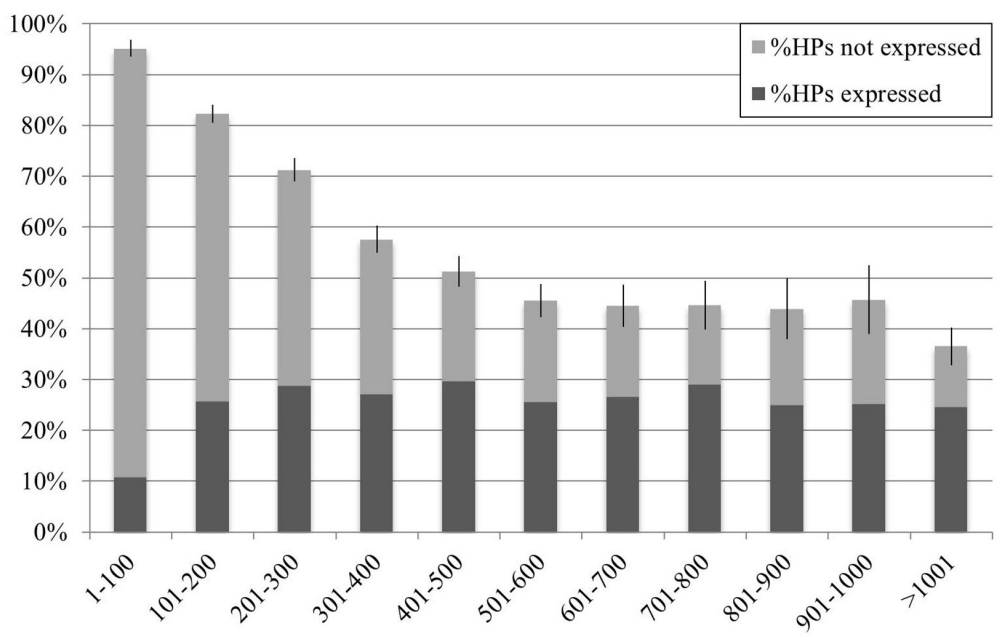

Figure 2. Length distribution of Paracoccidioides lutzii hypothetical proteins (HPs). The error bars correspond to the $95 \%$ confidence intervals.

\section{Targeting signals and characterized protein domains}

The predicted existence of targeting signals and specific domains in a protein suggests that it plays a functional role. Although these elements may also be found in pseudogenes as a result of gene duplication, their presence in a predicted amino acid sequence suggests that the protein is indeed expressed (Desler et al., 2009). Table 3 shows a summary of the predicted cellular localization of $P$. Iutzii HPs. Most appear to be localized in the cytoplasm (65 to $70 \%$ ), while around $20 \%$ contain a mitochondria-targeting peptide, and around $11 \%$ carry a secretory pathway signal peptide. The proportion of HPs allocated to each cellular compartment was similar between those identified by genome annotation and those expressed in the cDNA libraries. 
Table 3. Predicted cellular localization of Paracoccidioides /utzii hypothetical proteins according to the TargetP program.

\begin{tabular}{lcc}
\hline Predicted cellular localization & Number of HPs encoded in the Pb01 genome $(\%)^{*}$ & Number of HPs expressed in the cDNA libraries $(\%)^{* *}$ \\
\hline Mitochondrion & $1101(19.53 \%)$ & $525(22.21 \%)$ \\
Secretory pathway & $637(11.30 \%)$ & $275(11.63 \%)$ \\
Any other location & $3898(69.16 \%)$ & $1564(66.16 \%)$ \\
\hline
\end{tabular}

*Percentage based on the number of proteins annotated as HPs in the Pb01 genome (5636). **Percentage based on the number of unique HPs expressed (2364). HP = hypothetical protein; cDNA = complementary DNA.

Identifying conserved domains within a predicted polypeptide sequence can provide insight into the function, regulation, and/or localization of the putative protein. In total, $1984 \mathrm{HPs}$ in the $P$. lutzii genome included at least one domain (Table S2). In order to discern the biological implications of the HP domains identified in the cDNA libraries, we performed a functional enrichment analysis based on the best-represented protein family (Pfam) domains (Finn et al., 2014).

\section{Functional enrichment analysis}

Our functional enrichment analysis correlated the protein domains identified by Pfam with the expression of HPs in each condition (Table 4).

Table 4. Functional enrichment of the hypothetical proteins expressed under various conditions.

\begin{tabular}{|c|c|c|c|}
\hline Condition & Pfam term & Brief description & Corrected $\mathrm{P}$ value \\
\hline \multirow[t]{12}{*}{$M / Y$} & $\mathrm{zf}-\mathrm{CCHC}$ & DNA-dependent transcription regulation & 0.00 \\
\hline & Chromo & Structural domain associated with chromatin remodeling & 0.01 \\
\hline & BolA & Involved in the elongation and septation process during cell division & 0.19 \\
\hline & ChaC & Involved in active $\mathrm{Ca}^{2+} / \mathrm{H}^{+}$transport & 0.19 \\
\hline & SAP18 & Involved in RNA processing and apoptosis. & 0.19 \\
\hline & SRP19 & Involved in translation and translocation of proteins & 0.19 \\
\hline & SRPRB & Beta-subunit of the signal recognition particle receptor & 0.19 \\
\hline & DHFR_1 & Reduction of dihydrofolic acid to tetrahydrofolic acid, using NADPH as electron donor & 0.19 \\
\hline & Mit_rib_S27 & Involved in protein synthesis & 0.19 \\
\hline & Skp1_POZ & Involved in the ubiquitin protein degradation pathway & 0.19 \\
\hline & Ribosomal_S28e & Plays an important role in the elongation step of protein synthesis & 0.19 \\
\hline & Ubiq_cyt_C_chap & Required for assembly of coenzyme QF-2-cytochrome $\mathrm{C}$ reductase & 0.19 \\
\hline Transition & $\mathrm{zf}-\mathrm{CC} \mathrm{CH}$ & Transcription factor & 0.00 \\
\hline \multirow{2}{*}{ Infection } & $\mathrm{R} 3 \mathrm{H}$ & DNA-binding fator & 0.04 \\
\hline & Thioredoxin & Class of redox proteins, plays a role in response to reactive oxygen species & 0.18 \\
\hline
\end{tabular}

$\mathrm{M}=$ mycelium; $\mathrm{Y}=$ yeast; $\mathrm{Pfam}=$ protein family; $\mathrm{NADPH}=$ reduced nicotinamide adenine dinucleotide phosphate .

The functional domains overrepresented during the $M$ to $Y$ transition consisted of those involved in protein synthesis and regulation of gene expression, both by transcription factors and chromatin remodeling. The phase transition is a strictly regulated process resulting in cell shape change, alterations in cell wall composition, and several adjustments in protein expression (Felipe et al., 2005; Nunes et al., 2005; Klein and Tebbets, 2007). Although this dimorphic transition has been extensively studied, little is known of the cellular mechanisms orchestrating these events. Our findings suggest that HPs might play important roles in this process.

Domains related to the oxidative stress response were found in HPs expressed under conditions mimicking infection. Recent studies have shown that Paracoccidioides species respond strongly to the high concentrations of reactive oxygen species produced by cells of the host immune system (de Oliveira et al., 2013, Pigosso et al., 2013). 
Interestingly, domains involved in the regulation of gene expression were identified in all the conditions analyzed. This suggests that HPs are involved in reprogramming expression in response to a change in temperature, an event triggering differentiation, and the presence of hostderived factors.

\section{Functional classification}

$\mathrm{GO}$ is a functional classification scheme that defines, when possible, three categories for each gene product: biological process, molecular function, and cellular component (Ashburner et al., 2000). Of the $5636 \mathrm{HPs}, 3044$ were classified into at least one GO category (Figure 3 and Table S2). The HPs were implicated in a wide range of processes, including metabolism, establishment of localization, signaling, stimulus and stress responses, and cell wall organization and biogenesis. These results indicate that HPs take part in several cellular activities and may also participate in other, as yet undescribed, processes.

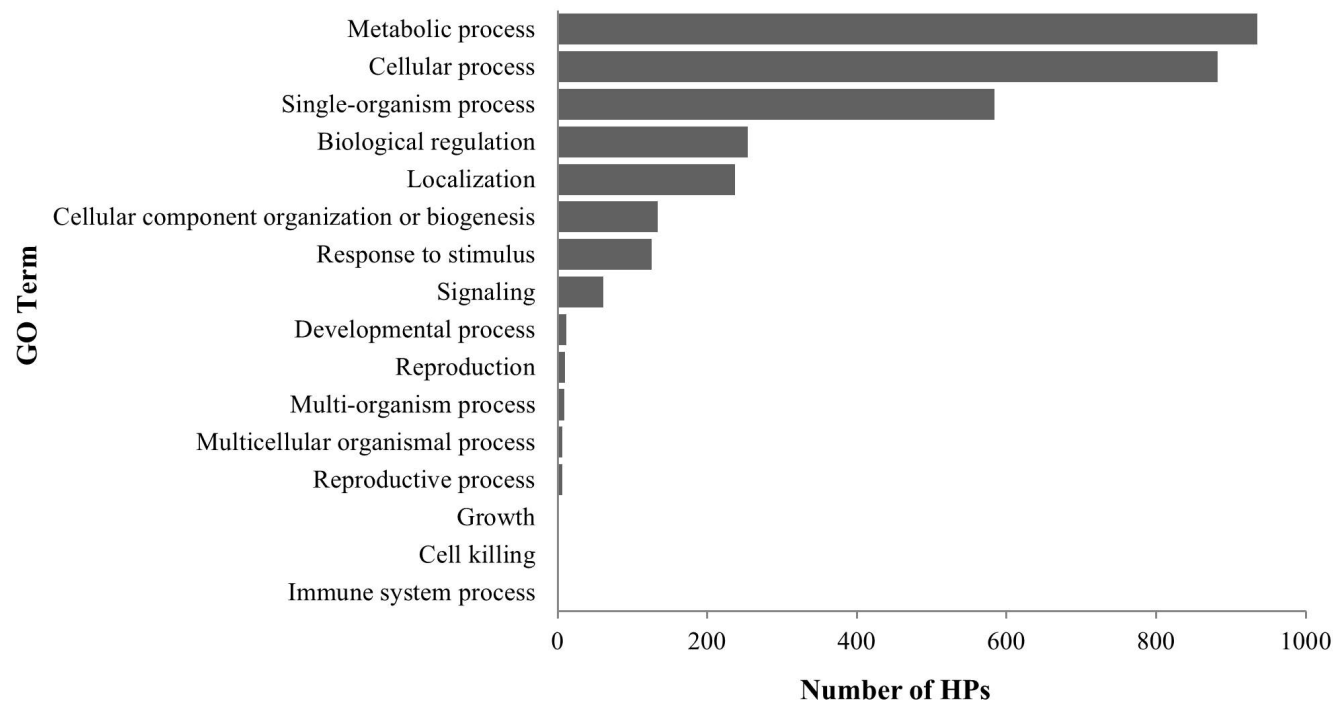

Figure 3. Functional classification of Paracoccidioides lutzii hypothetical proteins (HPs). GO = Gene Ontology.

\section{HP gene expression analysis using qPCR}

In order to verify their transcription profiles, qPCR assays (Figure 4) were performed for six randomly selected HP genes. All the genes tested were successfully amplified, demonstrating that they were indeed expressed. The qPCR results confirmed those obtained using the cDNA libraries, and in addition, revealed that some HPs were expressed under specific conditions but remained undetected in the corresponding library. For instance, the gene identified as PAAG_02090 was expressed in the $M$ to $Y$ transition, and that known as PAAG_08614 was expressed in $M$ and $Y$ states, as well as during transition, but neither were detected in any of the expression libraries. 


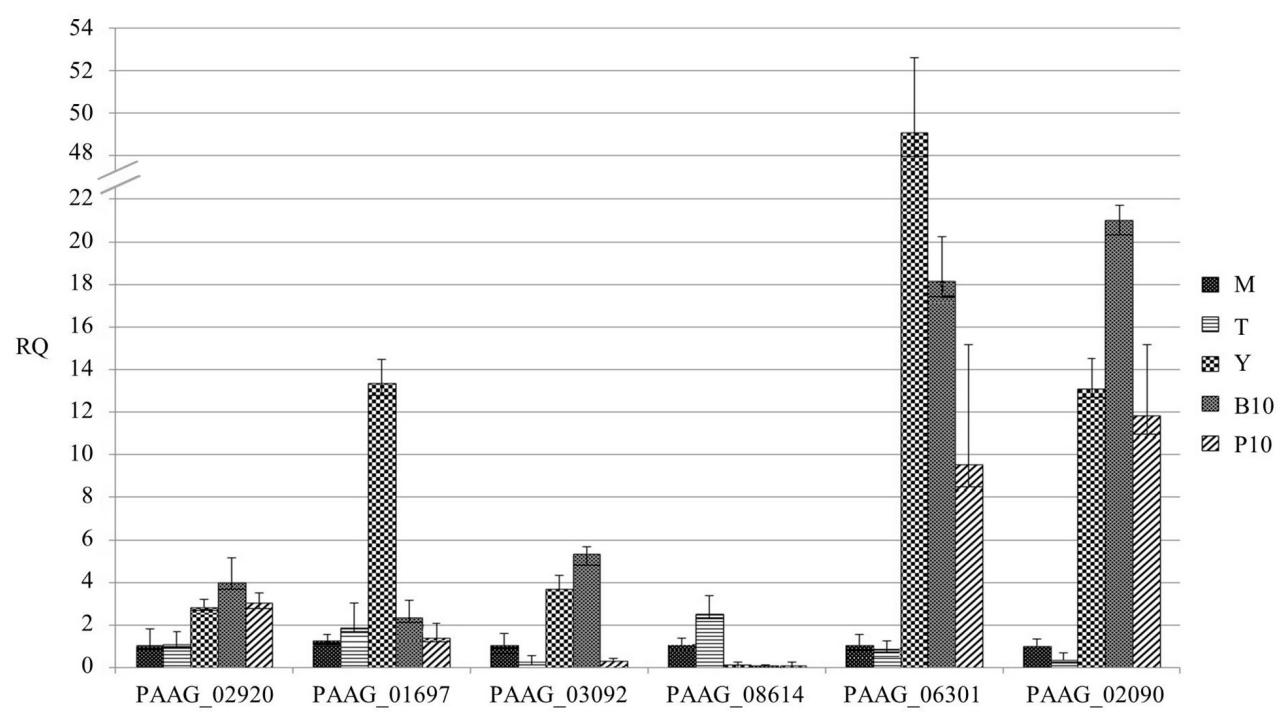

Figure 4. Transcriptional profiling of selected hypothetical proteins under different growth conditions. RNA samples were obtained from mycelial $(\mathrm{M})$ and yeast forms $(\mathrm{Y})$, cells undergoing transition ( $\mathrm{T} ; 48 \mathrm{~h}$ of $\mathrm{M}$ to $\mathrm{Y}$ transition), and $Y$ cells incubated with either human blood (B10) or plasma (P10). $R Q=$ relative quantity. The experiments were conducted in triplicate and the error bars represent standard deviations.

\section{CONCLUSIONS}

The large number of HPs encoded in the Paracoccidioides genome and expressed in its transcriptomes reveals a substantial gap in our knowledge regarding the functions of the proteins produced by these fungi. The characterization of such proteins constitutes a great challenge to our understanding of cellular metabolism and regulation of gene expression in microorganisms. This work highlights the significant contribution made by a large-scale computational analysis of $P$. lutzii HPs to the assignment of protein functions, and its ability to facilitate understanding of genomic and transcriptomic data.

\section{Conflicts of interest}

The authors declare no conflict of interest.

\section{ACKNOWLEDGMENTS}

Research supported by grants from Conselho Nacional de Desenvolvimento Científico e Tecnológico (CNPq - Edital Universal - Process \#478892/2011-0) and Fundação de Amparo à Pesquisa do Estado de Goiás (\#FAPEG Process \#201310267001074). P.F.F. Silva was supported by a fellowship from Coordenação de Aperfeiçoamento de Pessoal de Nível Superior (CAPES).

\section{Supplementary material}




\section{REFERENCES}

Altschul SF, Gish W, Miller W, Myers EW, et al. (1990). Basic local alignment search tool. J. Mol. Biol. 215: $403-410$. Ashburner M, Ball CA, Blake JA, Botstein D, et al. (2000). Gene ontology: tool for the unification of biology. The Gene Ontology Consortium. Nat. Genet. 25: 25-29.

Bailão AM, Schrank A, Borges CL, Dutra V, et al. (2006). Differential gene expression by Paracoccidioides brasiliensis in host interaction conditions: representational difference analysis identifies candidate genes associated with fungal pathogenesis. Microbes Infect. 8: 2686-2697.

Bailão AM, Shrank A, Borges CL, Parente JA, et al. (2007). The transcriptional profile of Paracoccidioides brasiliensis yeast cells is influenced by human plasma. FEMS Immunol. Med. Microbiol. 51: 43-57.

Bastos KP, Bailão AM, Borges CL, Faria FP, et al. (2007). The transcriptome analysis of early morphogenesis in Paracoccidioides brasiliensis mycelium reveals novel and induced genes potentially associated to the dimorphic process. BMC Microbiol. 7: 29.

Borges-Walmsley MI, Chen D, Shu X and Walmsley AR (2002). The pathobiology of Paracoccidioides brasiliensis. Trends Microbiol. 10: 80-87.

Conesa A, Götz S, García-Gómez JM, Terol J, et al. (2005). Blast2GO: a universal tool for annotation, visualization and analysis in functional genomics research. Bioinformatics 21: 3674-3676.

Costa M, Borges CL, Bailão AM, Meirelles GV, et al. (2007). Transcriptome profiling of Paracoccidioides brasiliensis yeast-phase cells recovered from infected mice brings new insights into fungal response upon host interaction. Microbiology 153: $4194-4207$.

de Oliveira MV, Oliveira AC, Shida CS, de Oliveira RC, et al. (2013). Gene expression modulation by paraquat-induced oxidative stress conditions in Paracoccidioides brasiliensis. Fungal Genet. Biol. 60: 101-109.

Desjardins CA, Champion MD, Holder JW, Muszewska A, et al. (2011). Comparative genomic analysis of human fungal pathogens causing paracoccidioidomycosis. PLoS Genet. 7: e1002345.

Desler C, Suravajhala P, Sanderhoff M, Rasmussen M, et al. (2009). In Silico screening for functional candidates amongst hypothetical proteins. BMC Bioinformatics 10: 289.

Doerks T, van Noort V, Minguez P and Bork P (2012). Annotation of the M. tuberculosis hypothetical orfeome: adding functional information to more than half of the uncharacterized proteins. PLoS One 7: e34302.

Emanuelsson O, Brunak S, von Heijne G and Nielsen H (2007). Locating proteins in the cell using TargetP, SignalP and related tools. Nat. Protoc. 2: 953-971.

Felipe MS, Andrade RV, Arraes FB, Nicola AM, et al. (2005). Transcriptional profiles of the human pathogenic fungus Paracoccidioides brasiliensis in mycelium and yeast cells. J. Biol. Chem. 280: 24706-24714.

Finn RD, Bateman A, Clements J, Coggill P, et al. (2014). The Pfam protein families database. Nucleic Acids Res. 42: 222-230

Franco M, Montenegro MR, Mendes RP, Marques SA, et al. (1987). Paracoccidioidomycosis: a recently proposed classification of its clinical forms. Rev. Soc. Bras. Med. Trop. 20: 129-132.

Goldman GH, dos Reis Marques E, Duarte Ribeiro DC, de Souza Bernardes LA, et al. (2003). Analysis of the human pathogen Paracoccidioides brasiliensis yeast phase: identification of putative homologues of Candida albicans virulence and pathogenicity genes. Eukaryot. Cell 2: 34-48.

ljaq J, Chandrasekharan M, Poddar R, Bethi N, et al. (2015). Annotation and curation of uncharacterized proteins- challenges. Front. Genet. 6: 119.

Klein BS and Tebbets B (2007). Dimorphism and virulence in fungi. Curr. Opin. Microbiol. 10: 314-319.

Livak KJ and Schmittgen TD (2001). Analysis of relative gene expression data using real-time quantitative PCR and the 2- $\triangle 4 \mathrm{Ct}$ method. Methods 25: 402-408.

Loewenstein Y, Raimondo D, Redfern OC, Watson J, et al. (2009). Protein function annotation by homology-based inference. Genome Biol. 10: 207.

Matute DR, McEwen JG, Puccia R, Montes BA, et al. (2006). Cryptic speciation and recombination in the fungus Paracoccidioides brasiliensis as revealed by gene genealogies. Mol. Biol. Evol. 23: 65-73.

Medoff G, Painter A and Kobayashi GS (1987). Mycelial- to yeast-phase transitions of the dimorphic fungi Blastomyces dermatitidis and Paracoccidioides brasiliensis. J. Bacteriol. 169: 4055-4060.

Nunes LR, Costa de Oliveira R, Leite DB, da Silva VS, et al. (2005). Transcriptome analysis of Paracoccidioides brasiliensis cells undergoing mycelium-to-yeast transition. Eukaryot. Cell 4: 2115-2128.

Pigosso LL, Parente AF, Coelho AS, Silva LP, et al. (2013). Comparative proteomics in the genus Paracoccidioides. Fungal Genet. Biol. 60: 87-100.

Qiao J, Shao M, Chen L and Wang J (2013). Systematic characterization of hypothetical proteins in Synechocystis sp. PCC 6803 reveals proteins functionally relevant to stress responses. Gene 512: 6-15.

Restrepo A (1985). The ecology of Paracoccidioides brasiliensis: a puzzle still unsolved. Sabouraudia 23: $323-334$.

Shahbaaz M, Hassan MI and Ahmad F (2013). Functional annotation of conserved hypothetical proteins from Haemophilus influenzae Rd KW20. PLoS One 8: e84263.

Teixeira MM, Theodoro RC, de Carvalho MJ, Fernandes L, et al. (2009). Phylogenetic analysis reveals a high level of speciation in the Paracoccidioides genus. Mol. Phylogenet. Evol. 52: 273-283. 